\title{
Pantographs and Phase Transitions for the Boundedness of Orbits
}

\author{
Evangelos Kranakis ${ }^{1,2, a)}$ Jorge Urrutia ${ }^{3,4, b)}$ \\ Received: January 6, 2020, Accepted: September 10, 2020
}

\begin{abstract}
We investigate the phase transition of a dynamical system generating a possibly infinite orbit of points. The points of the orbit are generated according to the following basic operation. Given a positive real number $a$, called the expansion factor, and two points $p, q$ at Euclidean distance $|p q|$ we determine the unique point $p^{\prime}$ on the straight line passing through $p$ and $q$ which is antipodal to the point $p$ with respect to $q$ and at a Euclidean distance $a|p q|$ from $q$. The operation on points previously defined is denoted by $p \Rightarrow_{a, q} p^{\prime}$. Let $\mathbf{a}:=a_{0}, a_{1}, \ldots, a_{n-1}$ be arbitrary but fixed positive real numbers and $\mathbf{q}:=q_{0}, q_{1}, \ldots, q_{n-1}$, be $n$ (anchor) points. An orbit consisting of an infinite sequence $p_{0}, p_{1}, \ldots, p_{m}, \ldots$ of points in the plane is generated by using the anchor points as follows. The orbit is initiated with an arbitrary point $p_{0}:=p$ and for all integers $m \geq 0$, satisfies $p_{m} \Rightarrow_{a_{m \bmod n}, q_{m \bmod n}} p_{m+1}$ so that $p_{m+1}:=\left(p_{m}\right)^{\prime}$. The resulting sequence of points is called the (a, q)-orbit of $p$. For any starting point $p$ and any pair $(\mathbf{a}, \mathbf{q})$ we characterize the boundedness of $(\mathbf{a}, \mathbf{q})$-orbits. Namely, we show that there is a phase transition concerning the boundedness of the resulting (a, q)-orbit which depends on whether the product $a_{0} a_{1} \cdots a_{n-1}$ of the expansion factors is less or larger than one. We also characterize the behaviour of the orbits when $a_{0} a_{1} \cdots a_{n-1}=1$. The "boundedness" phase transition phenomenon described above is shown to be valid for any dimension $d=1,2,3$ in Euclidean space. In addition, we propose variants of this approach for generating orbits on convex polygons, and propose several open problems corresponding to phase transition phenomena.
\end{abstract}

Keywords: Anchor Points, Boundedness, Line, Orbit, Phase Transition, Point

\section{Introduction}

We study a simple dynamical system defined by a procedure which is generating possibly infinite orbits of points by making use of an abitrary but fixed set of anchor points placed in Euclidean space. We investigate and characterize the boundedness of the orbits thus generated.

\subsection{Orbits from Anchor Points}

Before describing the problem we begin with some preliminary definitions and explanations of related concepts. Although the points and orbits generated may be in any dimension $d=1,2,3$ in Euclidean space, for the sake of simplicity in the discussion below we assume $d=2$. We indicate later how to transfer the methodology to all $d$.

Given a positive real number $a$, called the expansion factor, and two points $p, q$ at Euclidean distance $|p q|$ we determine the unique point $p^{\prime}$ on the straight line passing through the points $p$ and $q$ (see Fig. 1) which is antipodal to the point $p$ with respect to $q$ and at a Euclidean distance $a|p q|$ from $q$ (throughout this paper the notation $|\cdot|$ is used to define Euclidean distance).

Definition 1 The previously defined antipodal operation be-

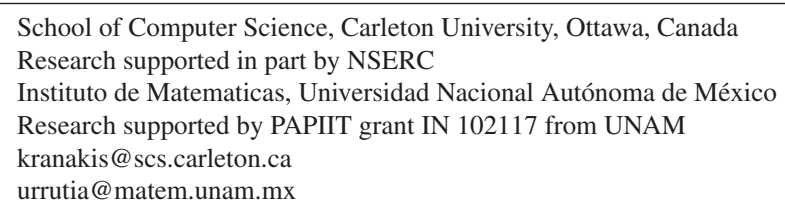

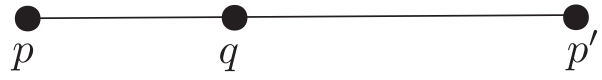

Fig. 1 The basic antipodal operation $p \Rightarrow_{a, q} p^{\prime}$ mapping a point $p$ to a unique point $p^{\prime}$ for generating orbits, where $a$ is the expansion factor at $q$ and $\left|q p^{\prime}\right|=a|p q|$.

tween points $p$ and $q$ with expansion factor $a>0$ is denoted by $p \Rightarrow_{a, q} p^{\prime}$.

Suppose that $n$ points $\mathbf{q}=\left(q_{0}, q_{1}, \ldots, q_{n-1}\right)$, called anchors are located in arbitrary but fixed positions in the plane.

The generated orbits consist of points forming trajectories in the plane. Assume that each point $q_{i}$ is associated with a positive real number $a_{i}$ called the expansion factor of $q_{i}$. Let $\mathbf{a}=$ $\left(a_{0}, a_{1}, \ldots, a_{n-1}\right)$ be the sequence of expansion factors. The consecutive points of the orbit are generated according to the basic antipodal operation described above as follows.

Definition 2 Let $p$ be any (starting) point in the plane. The $(\mathbf{a}, \mathbf{q})$-orbit of $p$ with respect to the sequence $\mathbf{q}$ of anchors consists of a possibly infinite sequence $p_{0}:=p, p_{1}, \ldots, p_{m}, \ldots$ of points which is generated by using the antipodal operation on the anchor points $q_{0}, q_{1}, \ldots, q_{n-1}$ so that for all $m \geq 0$ the point $p_{m+1}$ is antipodal to the point $p_{m}$ with respect to the anchor point $q_{m} \bmod n$ whose respective expansion factor is $a_{m \bmod n}$ (see Fig. 2).

The points of the orbit are generated by making use of the sequence of anchors $\mathbf{q}$ in the given order and repeats in that order "cyclically" in that after using the anchor $q_{n-1}$ it starts over with

A preliminary abstract of this work was presented by the first co-author in Ref. [1]. 


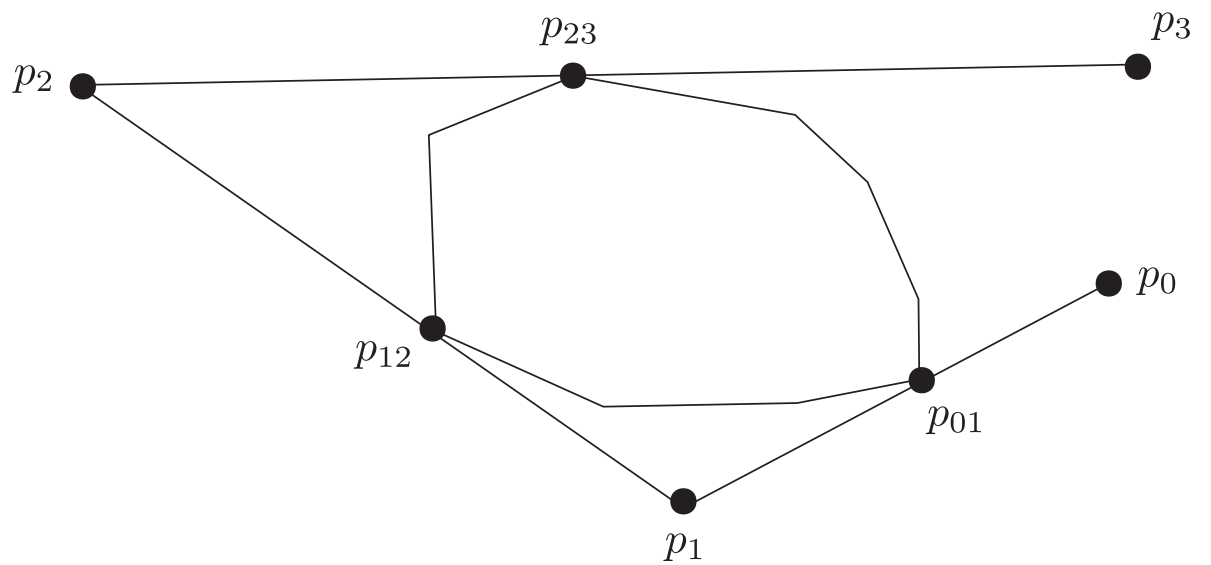

Fig. 3 The first three points $p_{0}, p_{1}, p_{2}, p_{3}$ in a $a$-forward outer billiard (orbit) for a bounded convex polygon with starting point $p_{0}$. By reversing direction $p_{2}, p_{1}, p_{0}$ this can also be considered a $a$-backward orbit.

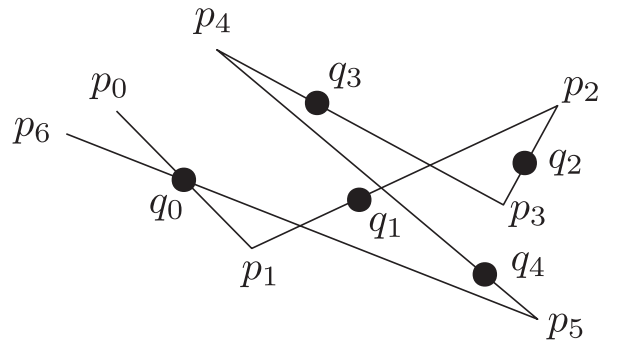

Fig. 2 The first seven points $p_{0}, p_{1}, p_{2}, p_{3}, p_{4}, p_{5}, p_{6}$ in a (a, $\left.\mathbf{q}\right)$-orbit for the unit segments with five anchor points $q_{0}, q_{1}, q_{2}, q_{3}, q_{4}$ in the plane and respective expansion factors $a_{0}, a_{1}, a_{2}, a_{3}, a_{4}$. The sequence of points $p_{i}$ continues indefinitely.

point $q_{0}$. The point $p_{m+1}$ is called the antipodal point of $p_{m}$ so that it satisfies $p_{m} \Rightarrow a_{m \bmod n}, q_{m \bmod n} p_{m+1}$. We use the simpler notation $p_{i} \Rightarrow p_{i+1}$, when the implied expansion factor $a_{i \bmod n}$ can be implied easily from the context. Iterating this operation there results an infinite sequence $p_{0} \Rightarrow p_{1} \Rightarrow p_{2} \Rightarrow \cdots$ (which may traverse a given point more than once) called the (a, q)-orbit of $p_{0}$ with respect to the pair $(\mathbf{a}, \mathbf{q})$, while $p_{0}$ is called the starting point of the resulting orbit (see Fig. 2).

Definition 3 The sequence of points thus generated from the starting point $p:=p_{0}$, are the anchor points $\mathbf{q}$ and the corresponding expansion factors $\mathbf{a}$ is denoted by $O_{p}(\mathbf{a}, \mathbf{q})$.

Unless it is necessary, mention of $\mathbf{q}$ may be omitted when this is easily implied from the context and when all the $a_{i}$ s are equal we then use the simpler notation $O_{p}(a)$, where we assume that $a_{0}:=a=\cdots=a_{n-1}$. Notice that if the anchor points are colinear and the starting point lies on this common line then the resulting orbit will also lie in its entirety on this line but otherwise in general the orbit will lie in the plane.

Definition 4 A sequence of points $\left\{p_{i}\right\}$ is said to be periodic if for some constant $k$ it satisfies $p_{i}=p_{i+k n}$, for $n=1,2, \ldots$.

Definition 5 An orbit $O_{p}(\mathbf{a}, \mathbf{q})$ is called bounded if it is a subset of some closed disk in the plane and unbounded, otherwise. Further, the (a,q)-orbits are unbounded (resp. bounded) if all non-periodic $O_{p}(\mathbf{a}, \mathbf{q})$ are unbounded (resp. bounded) for all points $p$.

Definition 6 An orbit $O_{p}(\mathbf{a}, \mathbf{q})$ is called closed if $p_{0}=p_{m+1}$, for some $m \geq 1$.

Closed orbits are clearlt periodic orbits. A closed orbit is also of course bounded.

Now we can formulate one of the main questions which will be studied in the remainder of this paper. (Note that the definitions and discussion above are valid for any dimension $d=1,2,3$ in Euclidean space.)

Problem 1 (Orbits from Anchor Points) Suppose that $n$ anchor points $\mathbf{q}=\left(q_{0}, q_{1}, \ldots, q_{n-1}\right)$ are located in the plane. Under what conditions on the real numbers $a_{0}, a_{1}, \ldots, a_{n-1}$ is the $(\mathbf{a}, \mathbf{q})$-orbit bounded or unbounded?

More specifically, we are interested in whether or not there is a phase transition concerning the boundedness of the orbits which depends on the product $a_{0} a_{1} \cdots a_{n-1}$.

\subsection{Orbits from Vertices of a Convex Polygon}

The orbit problem on point sets proposed in this paper is somewhat related to outer billiards (see Ref. [6]), that is a dynamical system defined in the Euclidean plane. This involves a discrete sequence of moves taking place outside a given bounded convex set $K$. The boundary of $K$ is a closed curve which may be smooth or polygonal. In addition, let $a$ be an arbitrary positive real number.

To form an outer billiard (orbit), we start with an arbitrary point $p:=p_{0}$ which lies outside the convex set $K$. We draw the straight line tangent to $K$ emanating from $p_{0}$ and intersecting $K$ at a vertex of $K$, for example denoted by $p_{01}$, so that $K$ is to the left of this line. Let $p_{1}$ be the point on this line antipodal to $p_{0}$ with respect to $p_{01}$ so that $\left|p_{0} p_{01}\right|=a\left|p_{01} p_{1}\right|$. Note that the point $p_{01}$ will be a vertex of the convex polygon which depends on the point $p_{0}$ and the convex polygon $K$.

Now iterate the same operation starting with the point $p_{1}$ leading to the segment $p_{1} p_{12} p_{2}$, and so on.

Definition 7 The sequence of points resulting when we iterate the previously described operation of drawing tangential line segments with expansion factor $a$ and which always keeps the convex polygon to their left (resp. right) called forward (resp. backward) $a$-orbit.

Figure 3 depicts an example of a forward outer billiard orbit with respect to a convex polygon starting from a point $p_{0}$.

It makes no difference whether one uses forward or backward $a$-orbits. For example, any finite backward orbit arises by revers- 
ing the direction of a finite forward orbit. For this reason, all $a$-orbits are considered forward $a$-orbits. Notice that an arbitrary orbit (outer billiard) may not necessarily traverse all the vertices of the convex polygon when rotating around the convex polygon and it may well traverse different vertices in another round ${ }^{* 1}$. Orbits from convex polygons will be visited again in Section 3 .

The definitions above are inspired from and are a generalization of analogous definitions restricted to $a=1$ and which can be found in the book [6] by Schwartz.

\subsection{Related Work}

Neumann [4] was the first to introduce outer billiards in the late 1950s. In the 1970s, Moser [3] popularized outer billiards as a toy model for celestial mechanics. More precisely, Moser [2][p.11] attributes the following question to Ref. [4]: Assume $a=1$.

"Is there an outer billiards system with an unbounded orbit?"

Moser [2] considers the above question as an idealized version of the problem of understanding the stability of the solar system.

For a book-length treatment of the topic as well as a chronological list of most known works related to the question of the boundedness of the resulting orbit when $a=1$ the reader is referred to the book by Schwartz [6]. We note that only outer orbits on Penrose kites are known to be unbounded [5]. To find general related work on billiards the reader is referred to the books [7], [8] by Tabachnikov.

There are certain similarities and differences between the problem of boundedness of orbits from anchor points and the polygonal case of outer billiard problem. There are similarities because they both generate orbits of points in the plane. There are also differences because of the way outer billiard orbits are generated. Given a convex polygon $K$, its sequence of vertices $\mathbf{q}=\left(q_{0}, q_{1}, \ldots, q_{n-1}\right)$ is also a sequence of anchor points. However an outer billiard (a, q)-orbit on $K$ is a trajectory that traverses these vertices cyclically but might skip some of them when it makes its next move since it requires that the next vertex is tangent to the polygon and this may not necessarily be the next vertex of the convex polygon in the natural cyclical order of the vertices on its perimeter. However, this is not the case in our problem on orbits for anchor points because we cannot skip any points in forming a trajectory.

To the best of our knowledge the phase transition phenomenon for the boundedness of orbits from anchor point sets considered in the present paper is new and has never been studied in the past.

\subsection{Outline and Results of the Paper}

For any set of anchors $\mathbf{q}=\left(q_{0}, q_{1}, \ldots, q_{n-1}\right)$ in the plane and associated expansion factors (positive real numbers) $\mathbf{a}=$ $\left(a_{0}, a_{1}, \ldots, a_{n-1}\right)$, we analyze and characterize the boundedness of the (a, q)-orbit. Theorem 1 is the main result in Section 2 and proves the phase transition in $d$-dimensional Euclidean space, for $d=1,2,3$, of the resulting orbit for any starting point $p$. Further, in Section 3 we look at similar questions for orbits on convex

*1 In a way, this is the main difference between orbits generated by the convex polygon with vertices $q_{0}, q_{1}, \ldots, q_{n-1}$ and the orbits generated from the anchor points $q_{0}, q_{1}, \ldots, q_{n-1}$. polygons and propose interesting open problems. Finally, Section 4 presents the conclusion.

\section{Orbits from Anchor Point Sets}

In this section we consider phase transitions for orbits arising from a set of anchor points. The main theorem to be proved in the sequel is the following.

Theorem 1 (Phase Transition on Boundedness of Orbits) Consider a sequence of $n$ anchor points $q_{0}, q_{1}, \ldots, q_{n-1}$ and associated expansion factors $a_{0}, a_{1}, \ldots, a_{n-1}$, respectively.

(1) Assume $a_{0} a_{1} \cdots a_{n-1} \neq 1$. Any $(\mathbf{a}, \mathbf{q})$-orbit visiting the points cyclically in the order $q_{0}, q_{1}, \ldots, q_{n-1}$ is either closed or else if the product $a_{0} \cdots a_{n-1}$ is greater than 1, it is unbounded, and if the product $a_{0} \cdots a_{n-1}$ is less than 1 it is bounded.

(2) Assume $a_{0} a_{1} \cdots a_{n-1}=1$.

(a) If $n$ is odd then any (a, q)-orbit visiting the points cyclically in the order $q_{0}, q_{1}, \ldots, q_{n-1}$ is periodic.

(b) If $n$ is even then any (a, q)-orbit visiting the points cyclically in the order $q_{0}, q_{1}, \ldots, q_{n-1}$ is either periodic or unbounded.

The statements above are equally valid for anchor points in $1 \mathrm{D}$, 2D and 3D space.

Details of the proof of Theorem 1 will be deferred until some basic concepts are introduced and necessary lemmas are proven.

The main ideas of the proof are as follows. In Section 2.1 we present the well-known concept of the pantograph leading to a simple methodology for deriving the first phase transition result presented in Section 2.2 and which is valid for $a_{0} a_{1} \cdots a_{n-1} \neq 1$. The phase transition for the case $a_{0} a_{1} \cdots a_{n-1}=1$ is analyzed in Section 2.3. We also prove the phase transition result in 1D, 2D and 3D space by applying the idea of projection. Finally, the proof of the main theorem will be completed in Section 2.4 by putting all these ideas together.

\subsection{Pantographs}

The approach of this section is inspired from the pantograph, a mechanical device used for copying and scaling in industrial design work. The pantograph is based on linkages of connected parallelograms so that the movement of one pen, in tracing an image, causes identical (up to scale) movements in another one pen or even more pens (see Ref. [9]). This simple principle of copying "up to scale" is founded on the simple Lemma 1 given below.

Lemma 1 (The Pantograph Lemma) Consider a sequence of $n$ anchor points $q_{0}, q_{1}, \ldots, q_{n-1}$ and associated expansion factors $a_{0}, a_{1}, \ldots, a_{n-1}$, respectively. Consider two different starting points $p_{0}, p_{0}^{\prime}$ and the resulting orbits $p_{0}, p_{1}, \ldots, p_{m-1}, \ldots$ and $p_{0}^{\prime}, p_{1}^{\prime}, \ldots, p_{m-1}^{\prime}, \ldots$ generated by the respective antipodal operations on these anchor points. If the point $p_{0}$ moves to the new point $p_{0}^{\prime}$ at distance $x$ from $p_{0}$ then the distance of the point $p_{i}$ from the point $p_{i}^{\prime}$ will be equal to $\left(a_{0} \cdots a_{i-1}\right) x$, for $1 \leq i \leq n$, respectively.

Proof. Since both transitions (see Fig. 4)

$$
p_{j-1} \Rightarrow_{a_{j-1}, q_{j-1}} p_{j} \text { and } p_{j-1}^{\prime} \Rightarrow_{a_{j-1}, q_{j-1}} p_{j}^{\prime}
$$

hold true, the triangles 


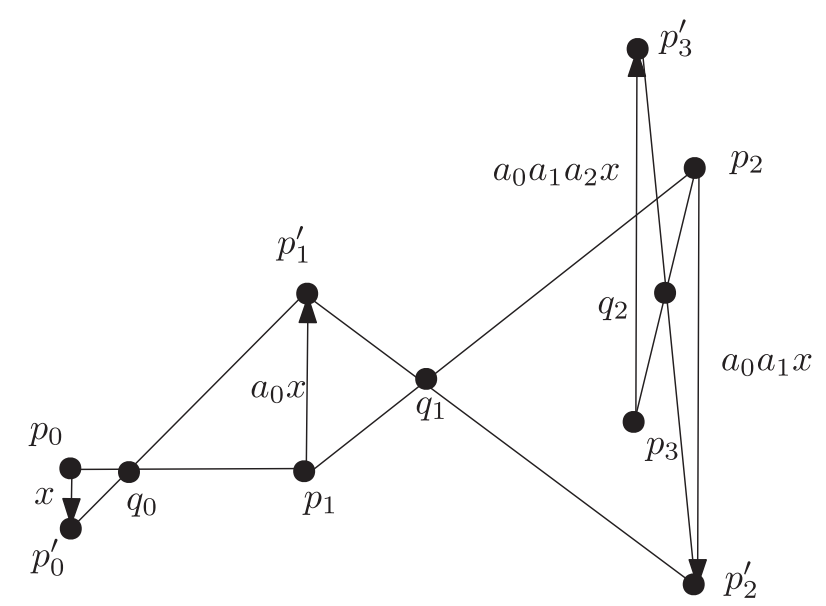

Fig. 4 Using similarity of triangles, it follows that if $\left|p_{0} p_{1}\right|=x$ then $\left|p_{0}^{\prime} p_{1}^{\prime}\right|=a \cdot x$.

$$
\triangle\left(p_{j-1} p_{j-1}^{\prime} q_{j-1}\right) \text { and } \Delta\left(p_{j} p_{j}^{\prime} q_{j-1}\right)
$$

must be similar, for all $j \geq 1$. Therefore the distance of the points $p_{i}$ and $p_{i}^{\prime}$ are as required, which proves Lemma 1 .

Notice that the drawing resulting from the original pen is proportional (similar) to the original drawing by a factor arising as a product of the expansion factors of the corresponding anchors.

As another useful corollary of Lemma 1 we note that if the point $p_{0}$ is moving towards the point $p_{0}^{\prime}$ tracing a smooth rectifiable curve $\gamma_{0}$ then the point $p_{j}$ will be moving towards the point $p_{j}^{\prime}$ tracing a smooth rectifiable curve $\gamma_{j}$ which can be obtained from $\gamma_{0}$ by uniformly scaling by a corresponding factor $a_{0} a_{1} \cdots a_{j}$, possibly with additional translation, rotation and reflection. Therefore, as can be seen easily from Fig. 4, if $p_{0}$ is moving towards $p_{0}^{\prime}$ along a straight line then also $p_{i}$ is moving towards $p_{i}^{\prime}$ (along a straight line). Moreover, the orientation of the curve is reflected, in the sense that if the expansion factor $\frac{\left|p_{j} p_{j}^{\prime}\right|}{p_{0} p_{0}^{\prime}}$ is equal to $a$, where $a>0$, and the curve $\gamma_{0}$ represents an arrow $\overrightarrow{p_{0} p_{0}^{\prime}}$ then the arrow $\overrightarrow{p_{j} p_{j}^{\prime}}$ corresponding to the curve $\gamma_{j}$ is reflected so as to satisfy

$$
\overrightarrow{p_{j} p_{j}^{\prime}}=\left\{\begin{array}{cl}
a\left(\overrightarrow{p_{0} p_{0}^{\prime}}\right), & \text { if } j \text { is even } \\
-a\left(\overrightarrow{p_{0} p_{0}^{\prime}}\right), & \text { if } j \text { is odd }
\end{array}\right.
$$

This property will turn out to be important in the proof of Lemma 3 which is used in the proof of the main Theorem.

\subsection{Phase Transition for $a_{0} a_{1} \cdots a_{n-1} \neq 1$}

Now we state and prove the existence of a phase transition phenomenon when $a_{0} \cdots a_{n-1} \neq 1$.

Lemma 2 (Phase transition when $a_{0} a_{1} \cdots a_{n-1} \neq 1$ )

Consider a sequence of $n$ anchor points $q_{0}, q_{1}, \ldots, q_{n-1}$ and associated expansion factors $a_{0}, a_{1}, \ldots, a_{n-1}$, respectively. Assume $a_{0} a_{1} \cdots a_{n-1} \neq 1$. Any $(\mathbf{a}, \mathbf{q})$-orbit visiting the points cyclically in the order $q_{0}, q_{1}, \ldots, q_{n-1}$ is either closed or else if the product $a_{0} \cdots a_{n-1}$ is greater than 1 , it is unbounded, and if the product $a_{0} \cdots a_{n-1}$ is less than 1 it is bounded.

Proof. Suppose that an orbit is started at a given point $p_{0}$, and after visiting the anchor points $q_{0}, q_{1}, \ldots, q_{n-1}$ cyclically in this order it ends up at a point $p_{n}$ using the sequence of consecutive antipodal operations as given below

$$
p_{0} \Rightarrow_{a_{0}, q_{0}} p_{1} \Rightarrow_{a_{1}, q_{1}} p_{2} \cdots p_{n-2} \Rightarrow_{a_{n-2}, q_{n-2}} p_{n-1} \Rightarrow_{a_{n-1}, q_{n-1}} p_{n}
$$

If $p_{n}=p_{0}$ then the orbit is closed (periodic).

Therefore without loss of generality we may suppose that $p_{0}$ is different from $p_{n}$ and let $d_{0}:=\left|p_{0} p_{n}\right|$ be the Euclidean distance between $p_{0}$ and $p_{n}$. Now continue the orbit starting from the point $p_{n}$ and by traversing the anchor points $q_{0}, q_{1}, \ldots, q_{n-1}$ one is ending at the new point $p_{2 n}$. By repeated application of the pantograph Lemma 1 mentioned above, if one traces the movement of the point $p_{0}$ to the point $p_{n}$, the point $p_{n}$ will move along the line joining $p_{0}$ to $p_{n}$ by a distance $d_{0} \cdot\left(a_{0} \cdots a_{n-1}\right)$. Moreover, the final position of $p_{n}$ will be $p_{2 n}$ (the position at which $p_{0}$ will end up after visiting all of the $q_{i}$ 's twice). If one visits all of the $q_{i}$ 's $k$ times, the distance between $p_{(k-1) n}$ and $p_{n k}$ will be $d_{0} \cdot\left(a_{0} \cdots a_{n-1}\right)^{k-1}$.

Now we can prove the phase transition claimed in the statement of the lemma. On the one hand, if the product $a_{0} \cdots a_{n-1}$ is bigger than one, then $\left|p_{(k-1) n} p_{k n}\right| \rightarrow \infty$, as $n \rightarrow \infty$. On the other hand, if $a_{0} \cdots a_{n-1}$ is less than one then using the triangle inequality we see that

$$
\begin{aligned}
\left|p_{k n} p_{0}\right| & \leq \sum_{i=1}^{k}\left|p_{(i-1) n} p_{i n}\right| \\
& =d_{0} \sum_{i=1}^{k}\left(a_{0} \cdots a_{n-1}\right)^{i-1} \\
& \leq d_{0} \frac{1-\left(a_{0} \cdots a_{n-1}\right)^{k}}{1-a_{0} \cdots a_{n-1}},
\end{aligned}
$$

which is bounded because $a_{0} \cdots a_{n-1}<1$.

To prove that the entire orbit is bounded we must employ a similar argument for the remaining points of the orbit. To this end, it is enough to define $d_{i}:=\left|p_{0} p_{i}\right|$, for $i<n$ and repeat the previous argument. This completes the proof of Lemma 2.

Lemma 2 shows that there is a phase transition for the orbits generated from a point set $q_{0}, q_{1}, \ldots, q_{n-1}$ of anchors with respective expansion factors $a_{0}, a_{1}, \ldots, a_{n-1}$, provided that $a_{0} a_{1} \cdots a_{n-1} \neq 1$. Next we look at the case $a_{0} a_{1} \cdots a_{n-1}=1$.

\subsection{Phase Transition for $a_{0} a_{1} \cdots a_{n-1}=1$}

Now we state and prove the existence of a phase transition phenomenon when $a_{0} \cdots a_{n-1}=1$.

Lemma 3 (Phase transition when $a_{0} a_{1} \cdots a_{n-1}=1$ )

Consider a sequence of $n$ anchor points $q_{0}, q_{1}, \ldots, q_{n-1}$ and associated expansion factors $a_{0}, a_{1}, \ldots, a_{n-1}$, respectively. Assume $a_{0} a_{1} \cdots a_{n-1}=1$.

(1) If $n$ is odd then any (a, q)-orbit visiting the points cyclically in the order $q_{0}, q_{1}, \ldots, q_{n-1}$ is periodic.

(2) If $n$ is even then any (a, q)-orbit visiting the points cyclically in the order $q_{0}, q_{1}, \ldots, q_{n-1}$ is either periodic or unbounded.

Proof. Suppose that an orbit is started at a given point $p_{0}$, and after visiting the anchor points $q_{0}, q_{1}, \ldots, q_{n-1}$ cyclically in this order it ends up at a point $p_{n}$ using the sequence of consecutive antipodal operations as follows

$$
p_{0} \Rightarrow_{a_{0}, q_{0}} p_{1} \Rightarrow_{a_{1}, q_{1}} p_{2} \cdots p_{n-2} \Rightarrow_{a_{n-2}, q_{n-2}} p_{n-1} \Rightarrow_{a_{n-1}, q_{n-1}} p_{n}
$$

If $p_{n}=p_{0}$ then the resulting orbit is closed (periodic). So without 

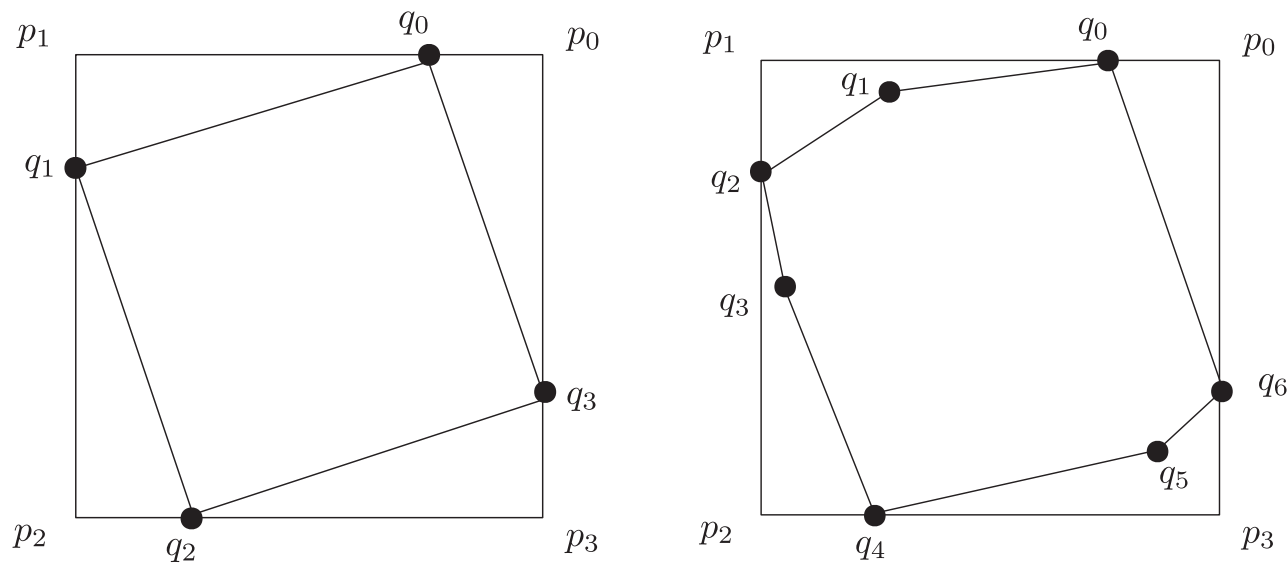

Fig. 6 Left picture: The four $q_{0}, q_{1}, q_{2}, q_{3}$ vertices of a square having the same expansion factor and a periodic orbit with four points $p_{0}, p_{1}, p_{2}, p_{3}$. Right picture: The seven vertices $q_{0}, q_{1}, \ldots, q_{6}$ of a convex polygon not having the same expansion factors and the resulting periodic orbit consisting of four points $p_{0}, p_{1}, p_{2}, p_{3}$.

loss of generality one can assume that $p_{n} \neq p_{0}$.

To complete the proof we argue as follows. As noted above, recall from Fig. 4 that every application of an antipodal operation reverses the direction of the arrow indicating the direction of movement from the point $p_{i}$ to the point $p_{i}^{\prime}$. Therefore the rest of the argument will depend on the parity of the index $n$.

Part 1. If $n$ is odd. Consider the next $n$ points $p_{n}, p_{n+1}, \ldots, p_{2 n-1}$ in the orbit of $p_{0}$ and the line segments $p_{0} p_{n}$ and $p_{n} p_{2 n}$. As a consequence of the pantograph Lemma 1 these segments are parallel and further $\left|p_{0} p_{n}\right|=\left|p_{n} p_{2 n}\right|$. Moreover, it follows from the observation above that the movement between the points $p_{0}$ and $p_{n}$ has opposite orientation from the movement between the points $p_{n}$ and $p_{2 n}$. As a result, $p_{2 n}=p_{0}$, which proves that the orbit is periodic.

Part 2. If $n$ is even. Consider the consecutive groups of $n$ points $p_{k n}, p_{k n+1}, \ldots, p_{(k+1) n-1}$ as generated by the antipodal operation which follows the anchor points $q_{0}, q_{1}, \ldots, q_{n-1}$ cyclically, for $k=0,1, \ldots$. As a consequence of the pantograph Lemma 1, the line segments $p_{k n} p_{(k+1) n}$, for $k-0,1, \ldots$ are parallel and of equal length. Since $\left|p_{k n} p_{(k+1) n}\right| \neq 0$, as a consequence of the pantograph Lemma 1, these are parallel and further $\left|p_{0} p_{n}\right|=\left|p_{n} p_{2 n}\right|$. Moreover, it follows from the observation above that the movement between the points $p_{k n}$ and $p_{(k+1) n}$, for all $k$, has the same orientation. As a consequence, the resulting orbit is unbounded.

This completes the proof of Lemma 3.

\subsection{Proof of Theorem 1}

Proof. Now we are in a position to complete the details of the proof of Theorem 1 . The results proven so far are equally valid in $1 \mathrm{D}$ with the interpretation that the anchor points lie on a straight line and the starting points of the orbits are placed on this line.

We can extend the result to anchor points in space by projecting the anchor points to points of an infinite plane so that different points in 3D are projected to different points in 2D (see Fig. 5). The projection clearly maintains the same expansion factors for the projected points. Additional details required to complete the proof are left up to the reader.

By combining Lemmas 2 and 3 together with the above observation on projecting anchor points the proof of Theorem 1 is

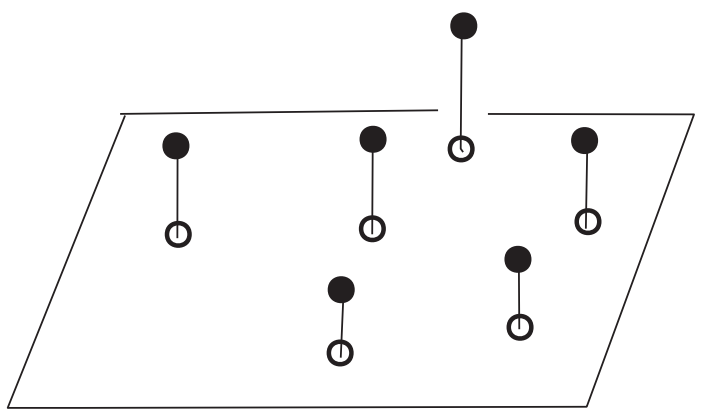

Fig. 5 A set $q_{0}, q_{1}, \ldots, q_{n-1}$ of $n$ anchors in 3D space. Each anchor $q_{i}$ is projected to the infinite plane.

easily completed.

\section{Orbits from Convex Polygons}

Consider a convex polygon with vertices $q_{0}, q_{1}, \ldots, q_{n-1}$ in the counterclockwise order (see Fig. 3). Each vertex $q_{i}$ is associated with a positive real number $a_{i}$, called the expansion factor. To form an outer billiard orbit, one starts with an arbitrary point $p_{0}$ which lies outside the convex polygon $K$ and draws a straight line tangent to $K$ emanating from $p_{0}$ and intersecting $K$ at a vertex so that $K$ is to the left of this line. Let $p_{1}$ be the point on this line antipodal to $p_{0}$ with expansion factor $a_{0}{ }^{* 2}$. Now iterate the same operation starting with the point $p_{1}$ so that $p_{2}$ is the point antipodal to $p_{1}$ and expansion factor $a_{1}$, leading to the segment $p_{1} p_{2}$, and so on. See Fig. 3 which depicts an $\left(a_{0}, a_{1}, \ldots, a_{n-1}\right)$ orbit (outer billiard) generated from a starting point $p_{0}$.

Example 1 Consider the example depicted in Fig. 6 below. The left side of Fig. 6 depicts a periodic orbit with four points $p_{0}, p_{1}, p_{2}, p_{3}$ having identical expansion factors arising from a square with four vertices $q_{0}, q_{1}, q_{2}, q_{3}$. The right side of Fig. 6 depicts a periodic orbit consisting of four points $p_{0}, p_{1}, p_{2}, p_{3}$ for a convex polygon with seven vertices $q_{0}, q_{1}, \ldots, q_{6}$ not having the same expansion factors.

The example just described indicates the main difficulties with characterizing phase transition phenomena for orbits arising from

*2 If the tangent is parallel to an edge then by convention we select as antipodal point the first vertex of the convex polygon intersected by this line. 


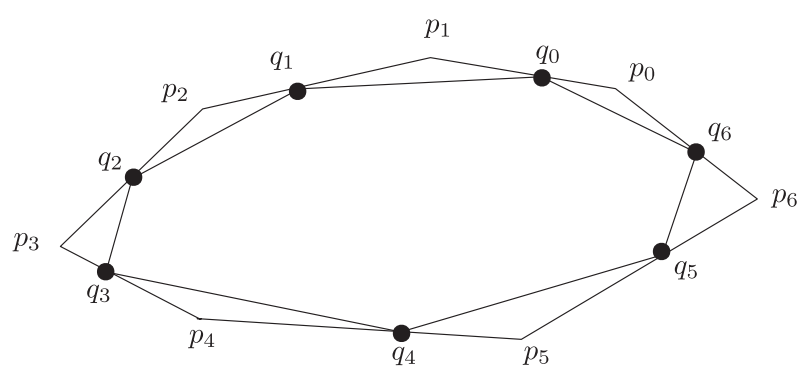

Fig. 7 A convex polygon with 7 vertices $q_{0}, q_{1}, \ldots, q_{6}$ and a periodic outer billiard $p_{0}, p_{1}, \ldots, p_{6}$ in an outer billiard (orbit) for a convex polygon with starting point $p_{0}$.

convex polygons. First of all we must restrict our attention to nonperiodic orbits. Second, even for non-periodic orbits one must take into account the fact that (non-)periodic orbits may omit vertices of the convex polygon.

Therefore, unlike the case of orbits from anchor point sets already analyzed in Section 2 it is not a priori obvious that a condition on the size of the product $a_{0} a_{1} \cdots a_{n-1}$ can ensure a phase transition on the boundedness of the resulting orbits. Therefore the following question (which is similar to question posed in Problem 1) is left as an open problem.

Problem 2 (Phase transition on convex polygons) Suppose we are given a convex polygon with vertices $q_{0}, q_{1}, \ldots, q_{n-1}$ and associated expansion factors, $a_{i}$, for $i=0,1,2, \ldots, n-1$ respectively. Is there a phase transition for the boundedness of non-periodic orbits which depends on the value of the product $a_{0} a_{1} \cdots a_{n-1}$, in other words, if $a_{0} a_{1} \cdots a_{n-1}<1$ then the orbit generated is bounded and if $a_{0} a_{1} \cdots a_{n-1} \geq 1$ then the orbit generated is unbounded?

This problem subsumes the main question on the boundedness of outer billiards initially posed by Neumann [4] and popularized by Moser [3], already mentioned in the related work in Section 1.3. Therefore it is not expected to be easy.

In general we can prove the following theorem.

Theorem 2 For any sequence of $n$ expansion factors $a_{0}, a_{1}, \ldots, a_{n-1}$ there esist a sequence of $n$ points $q_{0}, q_{1}, \ldots, q_{n-1}$ forming a convex polygon and a periodic orbit $p_{0}, p_{1}, \ldots, p_{n-1}, p_{n}=p_{0}$ visiting all the vertices of the convex polygon in their given order such that

$$
\frac{\left|p_{i} q_{i}\right|}{\left|q_{i} p_{i+1}\right|}=a_{i}, \text { for all } i=0,1, \ldots, n-1 \text {. }
$$

Proof. The proof is depicted in Fig. 7. Start with a sequence of points $p_{0}, p_{1}, \ldots, p_{n-1}, p_{n}=p_{0}$ forming a convex polygon. On each line segment $p_{i} p_{i+1}$ select a point $q_{i}$ so that

$$
\frac{\left|p_{i} q_{i}\right|}{\left|q_{i} p_{i+1}\right|}=a_{i}
$$

for all $i=0,1, \ldots, n-1$. Clearly, the points points $q_{0}, q_{1}, \ldots, q_{n-1}$ form a convex polygon. Moreover, the orbit associated with the convex polygon $q_{0}, q_{1}, \ldots, q_{n-1}$ and emanating from the point $p_{0}$ is periodic, by construction. This completes the proof of Theorem 2.

\section{Conclusion}

In this paper we introduce a new dynamical system defined in the Euclidean plane or space and study its phase transition for point sets. For any sequence of $n$ positive real numbers $\mathbf{a}=\left(a_{0}, a_{1}, \ldots, a_{n-1}\right)$, we analyze and characterize the boundedness of the resulting (a, $\mathbf{q})$-orbits, where $\mathbf{q}=\left(q_{0}, q_{1}, \ldots, q_{n-1}\right)$ is a corresponding arbitrary sequence of $n$ anchors in the plane. In particular, we prove that there is a phase transition concerning the boundedness of an $(\mathbf{a}, \mathbf{q})$-orbit which depends on whether or not $a_{0} a_{1} \cdots a_{n-1}<1$. We also propose several related problems for orbits arising from considering collections of anchor points forming a convex polygon.

\section{References}

[1] Kranakis, E.: A Phase Transition Concerning the Boundedness of Orbits on a Point Set (2 Page Abstract), Japanese Conference on Discrete and Computational Geometry, Graphs and Games (JCDCGGG) (2019).

[2] Moser, J.: Stable and random motions in dynamical systems: With special emphasis on celestial mechanics (AM-77), Princeton University Press (1973).

[3] Moser, J.: Is the solar system stable?, The Mathematical Intelligencer, Vol.1, No.2, pp.65-71 (1978).

[4] Neumann, B.H.: Sharing ham and eggs, Iota, Manchester University, pp.14-18 (1959).

[5] Schwartz, R.E.: Unbounded orbits for outer billiards I, Journal of Modern Dynamics, Vol.1, No.3, pp.371-424 (2007).

[6] Schwartz, R.E.: Outer Billiards on Kites (AM-171), Princeton University Press (2009).

[7] Tabachnikov, S.: Billiards, Panoramas et Synthèses, Vol.I, Société Mathématique de France, "Panoramas et Syntheses" (1995).

[8] Tabachnikov, S.: Geometry and Billiards, Student Mathematical Library, Vol.30, American Mathematical Society (2005).

[9] Wikipedia: Pantograph, available from 〈https://en.wikipedia.org/wiki/ Pantograph $>$ (accessed 2019-08-07).

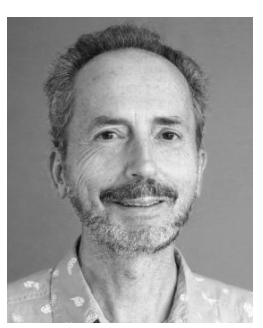

Evangelos Kranakis received a B.Sc. in Mathematics from the University of Athens, Greece, in 1973 and a Ph.D. also in Mathematics from the University of Minnesota, USA, in 1980. From 1980 to 1991 he held various faculty positions in Purdue University, USA, University of Heidelberg, Germany, Yale University, USA, Universiteit van Amsterdam, and Centrum voor Wiskunde en Informatica (CWI) in The Netherlands. He joined the faculty of the School of Computer Science of Carleton University, Ottawa, Canada, in the Fall of 1991. His current research interests include Algorithmics, Distributed and Computational Biology, Distributed and Mobile Agent Computing, Networks (Ad Hoc, Communication, Sensor, Social), and Cryptographic and Network Security. 


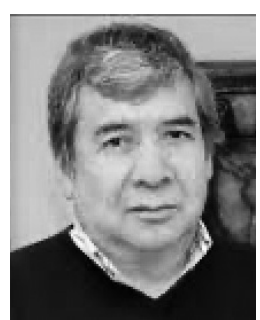

Jorge Urrutia is Professor of Mathematics at UNAM in Mexico City. He received his Ph.D. from the University of Waterloo and was a member of the faculty of Computer Science at the University of Ottawa, in Canada. He was co-founding editor, and co-editor-in-chief (until Dec. 1999) of the journal Computational Geometry, Theory and Applications published by Elsevier. He is currently in the editorial board of member of the editorial boards of Computational Geometry, Theory and Applications, Graphs and Combinatorics. 